\title{
Neural Network Method for Inverse Modeling of Material Deformation
}

\author{
Nenad Ivezic, John D. Allen Jr., and Thomas Zacharia \\ Computer Science and Mathematics Division \\ Oak Ridge National Laboratory \\ Oak Ridge, TN 37831, USA
}

\begin{abstract}
A method is described for inverse modeling of material deformation in applications of importance to the sheet metal forming industry. The method was developed in order to assess the feasibility of utilizing empirical data in the early stages of the design process as an alternative to conventional prototyping methods. Because properly prepared and employed artificial neural networks (ANN) were known to be capable of codifying and generalizing large bodies of empirical data, they were the natural choice for the application. The product of the work described here is a desktop ANN system that can produce in one pass an accurate die design for a user-specified part shape.
\end{abstract}

\section{INTRODUCTION}

Sheet metal forming applications have traditionally involved costly, iterative, and time-consuming design methodologies based on physical prototyping. Recently, finite element analysis techniques have been utilized with varying success for modeling and prediction of sheet metal deformation processes during the design stages [1]. Additionally, certain methods from the field of artificial intelligence (AI) have been brought to bear on the problem of automating the design of tooling for the manufacture of metal stampings [2]. Yet ANN methods, among the most powerful AI tools, have been but scarcely explored in this context, although they have been used to solve engineering design problems that involved identification, learning, and prediction of scalar or vector design quantities $[3,4,5]$. In contrast, the method described in this paper addresses the problem of capturing deformation relationships governing three-dimensional mapping in sheet metal stamping applications. Presented results demonstrate that the inverse of these relationships may be captured and used to predict the die geometry that will, under specified material and stamping conditions, lead to the production of a user-specified part shape.

\section{DEVELOPMENT OF THE METHOD}

Realization of the ANN-based die design tool depended critically on the successful solution of several inter-related problems. Central to all of these was the development of a suitable method for representing die and part shapes to the input nodes of a neural network (or networks, when multiple networks were employed). For network training, these data would be of two classes. Input data would represent the conformation of the part for which a die geometry was sought. Output data (those data applied to the output node(s) to specify to the network the result that should obtain for the corresponding input) would represent in some manner the die geometry that the network should learn to produce in response to the input data. Although it is the case, in principle at least, that a network should be able to learn in the context of almost any representation, relatively simple (and not always obvious) variations in the representation scheme can produce striking differences in the efficiency with which the learning process takes place. Described below are several of the surface-representation methods investigated during development of the die prediction system.

Simplest in concept, but surely least effective of all, was a direct mapping of part and die data onto the input and output nodes of a very large network. Here, the input and output nodes were arranged in "rectangular" fashion, the number of each node class being equal to the size of the part/die meshes (never more than 25 by 25 for this first attempt). Although this method worked adequately for some initial demonstrations of the general technique, it was doomed to failure by the impossibility of obtaining enough training data to condition the network properly. Even had data been available in sufficient quantity, it is doubtful that any single network architecture would be capable of generalizing the shape transformation relationships over a useful spectrum of part shapes under such a 


\section{DISCLAIMER}

This report was prepared as an account of work sponsored by an agency of the United States Government. Neither the United States Government nor any agency thereof, nor any of their employees, make any warranty, express or implied, or assumes any legal liability or responsibility for the accuracy, completeness, or usefulness of any information, apparatus, product, or process disclosed, or represents that its use would not infringe privately owned rights. Reference herein to any specific commercial product, process, or service by trade name, trademark, manufacturer, or otherwise does not necessarily constitute or imply its endorsement, recommendation, or favoring by the United States Government or any agency thereof. The views and opinions of authors expressed herein do not necessarily state or reflect those of the United States Government or any agency thereof. 


\section{DISCLAIMER}

Portions of this document may be illegible in electronic image products. Images are produced from the best available original document. 
representational scheme. If it could do so at all, network prediction performance would be very sensitive to minor displacements of presented shapes relative to the input grid.

A method based on two-dimensional Fourier transforms derived its utility from the fact that a relatively small fraction of the total number of Fourier components (of the order of ten percent) could represent a three dimensional shape with considerable fidelity. As employed for representing die and part geometries to a neural network, the transform was applied to each of the data sets to be presented for evaluation. The training goal for the network was taken to be the development of the functional relationships linking the Fourier components representing a part configuration (together with the associated data defining material and forming parameters) with the corresponding Fourier components representing the die that would, under the specified set of material and forming parameters, be suitable for producing that part. When a fully trained Fourier-based network is presented with a data set representing a part (and the associated material and forming parameters) for which a die design is required, the network produces at its output nodes a set of values representing the (scaled) Fourier components of the desired die. Recovery of the die specification requires only an appropriate renormalization and retransformation into a Cartesian coordinate representation.

A somewhat similar method based on two-dimensional Wavelet transforms was developed as the next step in the search for an efficient representation method for surface data. The 2D Wavelet method, like the Fourier method described above, derives its utility from the fact that a very small fraction of the total number of wavelet components (perhaps one or two percent) can represent a surface with considerable fidelity. For a reasonably narrowly defined set of shapes, it is observed that the identity of the important Wavelet components is fairly consistent across all members of the set with variations among set members being represented principally in amplitude differences among the important components. Substantive variations among set members will be represented by the appearance of dominant wavelet components more or less singular to the set members bearing those variations. It is the combination of consistent and singular components that is captured and represented to the network for subsequent processing.

Although the Two Dimensional Wavelet representation method reduced the number of data required for shape representation by a greater factor than that obtaining for the Two Dimensional Fourier method, the reduction still appears insufficient to support completely general network training. The single greatest advantage is one of speed. Once trained, the network executes in milliseconds on any reasonably modern desktop computer.

The most successful of the investigated methods for die/part representation is the so-called Weighted Patch method. Development of this scheme was predicated on the assumption that shape gradients near a point on a material surface are more likely to be predictive of the response of material at that point to deformation forces (imposed, for instance, by a punch and die) than are shape gradients at positions more removed from the point. Equally reasonable is the assertion that "near" effects must be represented more completely to the neural network than more "distant" ones if the network is to capture important metal forming relationships.

In the Weighted Patch Method, a fixed pattern of "averaging regions" is scanned over the data array representing a part for which a die is to be developed. In a typical implementation, there may be 19 averaging regions of graduated sizes ( 9 for each of two orthogonal axes and one central element, the shortest being one array element square, the longest of the order of 8 to 10 array elements in length by one element in width) comprising a cross shaped "Patch Geometry" of the general form suggested by the schematic representation of Figure 1. These averaging regions are distributed along orthogonal axes (axes typically parallel the length and breadth of the part). Each averaging region is represented at the network input layer by a single node.

The training goal for a Patch Method network is to produce, for each element of the part array, the value of the difference between part elevation and corresponding die elevation at that point. When the network is fully trained a predicted die configuration can be trivially derived from these differences for any presented part shape.

The Patch Method confers the advantage that a somewhat more accurate representation of the part surface is retained in the reduced data employed by the network than is the case with either the Fourier or Wavelet data representation methods. Perhaps more important than this is the fact that, since each point on a surface is treated as 
a separate training example, the network can develop a much better codification of relationships linking part and die shape than can a network employed for either of the previously discussed methods.

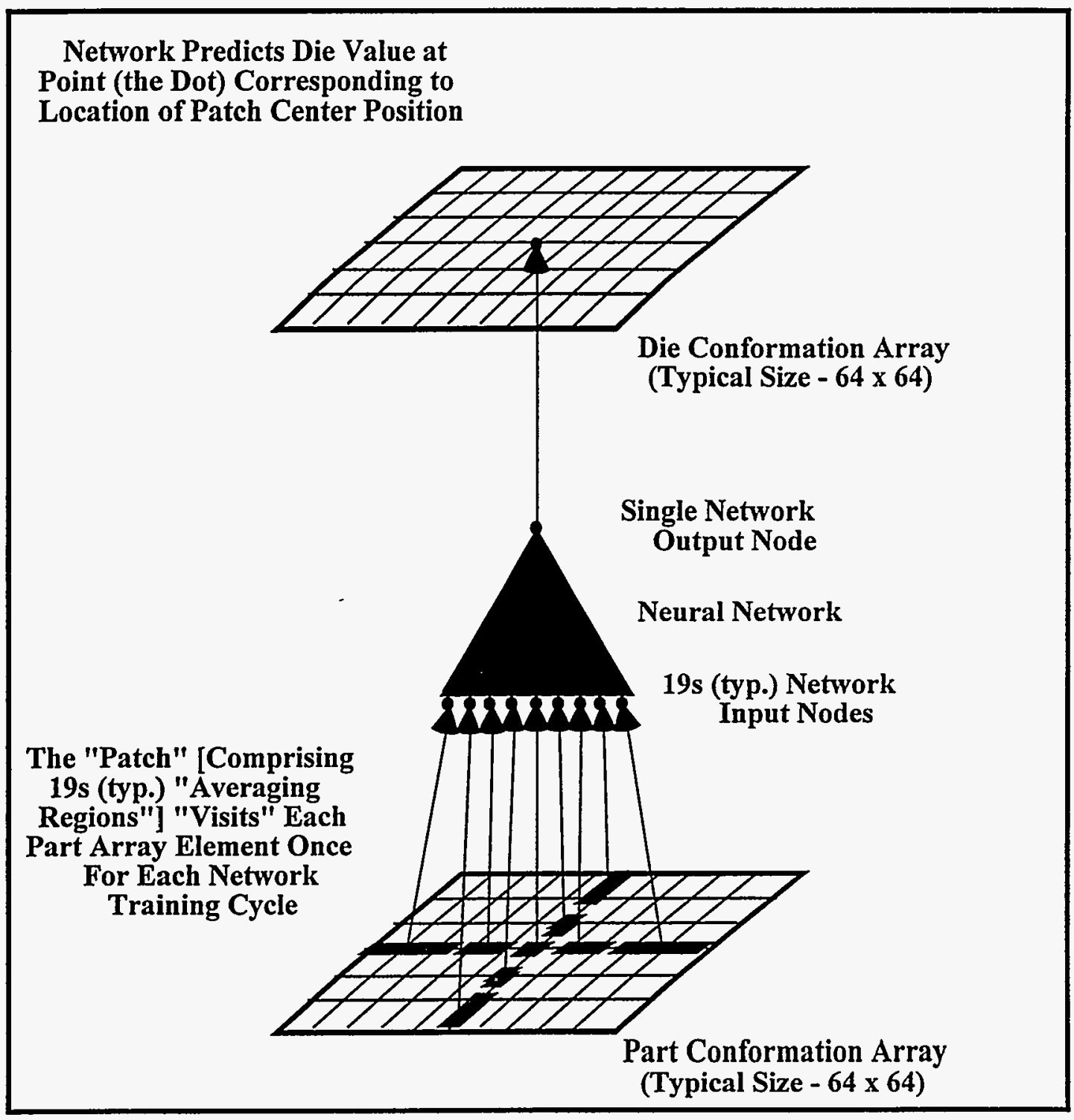

Fig. 1. Patch method schematic diagram (parameter nodes are not shown)

The penalties paid for realizing these advantages are two in number. First is the requirement that the network "visits" each of the elements of the part definition array many times during training. For the $64 \times 64$ data sets employed to develop the results presented here, there are 4096 such elements, each of which may be visited several hundred times during a typical training session. The second penalty is the memory intensive character of the method. For each of the 4096 potential patch-center locations, it is necessary to store the 19 values comprising the Patch (for a total of $19 * 4096$, or 77824 values) for each data set to be processed by the network (of which data sets there may be hundreds).

\section{EXPERIMENTAL DEMONSTRATION}

The potential power of the Weighted Patch method is well illustrated by the results of a simple experimental demonstration, for which the goal was to demonstrate that a network trained under the method could generalize its training results well enough to produce a die design for a member of a shape class to which it had not been exposed during training. 
For this demonstration, part definitions were created from corresponding die definitions by a relatively simple deformation model that included two variable parameters, one representing a material property, the other a forming variable. The characteristics of the model were chosen to ensure that the resulting shape transformations would be of sufficient complexity to challenge the network.

Model output was derived from die representations of the three generic shapes depicted in Figure 2. For each die shape class, variation of the relevant defining parameters resulted in a spectrum of die geometries from which corresponding parts would be "formed" by the deformation model. Pan dies, for instance, were defined by mean base width, mean base height, mean top width, mean top height, top and bottom aspect ratios (so that either base, top, or both could assume trapezoidal shape), center elevation, and top tilt angle. Additional parameters specified the presence or absence of ridges and their spatial extents relative to the base die dimensions. Similar parameters (with the addition of eccentricity and appropriate omissions) defined the model Top Hat and Ellipsoidal Shell dies. The value of one last variable determined the extent to which die corners were rounded. In all cases, the die elevations were defined on a regular, 64 by 64 element, square grid. Although this representation may at first appear contrary to the conventions of standard deformation modeling, it is entirely in keeping with the metrological considerations that would dictate the collection of data from physical die/part pairs.

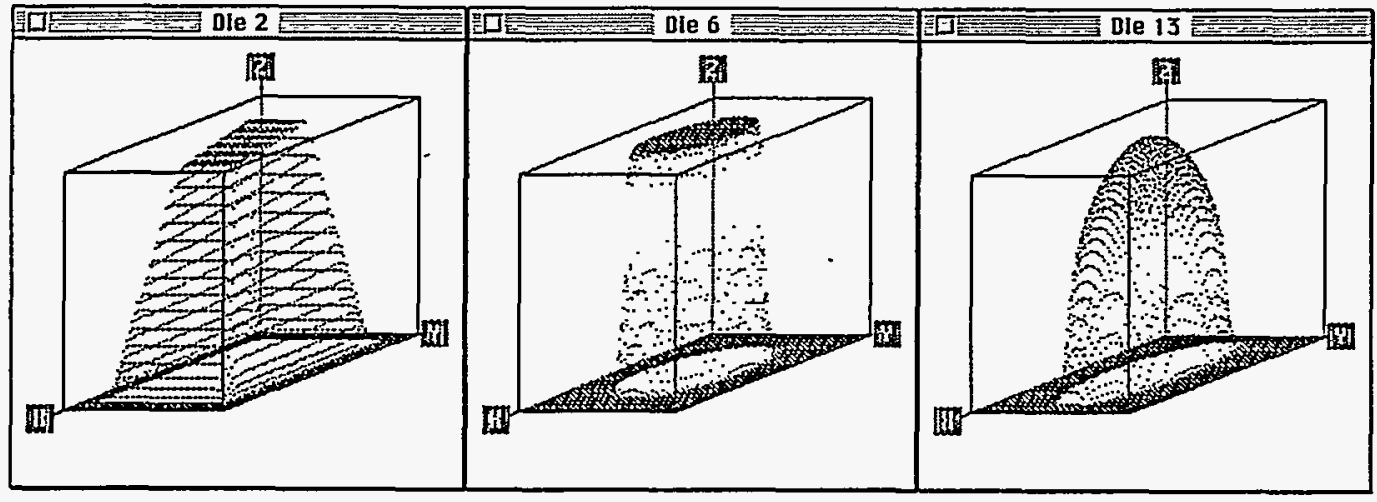

Fig. 2. Pan, TopHat, and Shell Die Shapes

The simple deformation model created part data from each of the die representations as a parameterized displacement function of the local and the global geometries of the die. It should be noted that, although the simple deformation model calculates material displacements in each of the three axes, the final part data are interpolated back onto a regular 64 by 64 grid. These, expressed as an array of elevations, are stored with the generating die specification (again, an array of elevations) and a list of the "material" and "forming" parameters associated with the part/die pair to form a single training data set.

Network training involved 40 part/die pairs. Of these, 20 defined variations on the Pan geometry. The remaining 20 defined variations on the Elliptical Shell geometry. For the Pan parts, elevation, aspect ratio, top tilt, and top and bottom shape (square or trapezoid) were varied. Elevation values (normalized to mean base size) fell in the range $(.062, .125)$. Mean top size to mean base size ratio varied between 0.5 and 1.0 . Aspect ratio (effectively mean length to mean width) varied over the range $(.47,1.0)$. Top tilt angle values (in radians) fell in the range $(0.0,0.5)$. Where they were relevant, the same parameter ranges characterized the generated Elliptical Shell part/die pairs. Parameters of the deformation model were established to limit elevation differences between die and predicted part to no more than about ten percent.

Pre-processing of data included the interpolation and normalization steps required to produce the 4096 input Patch vectors and the corresponding output value for each of the 40 training data sets and the single TopHat test data set. Node components and output values for the training data were normalized to the range $(-.85,+.85)$. Postprocessing to recover the die shape involved an inversion of the output normalization process and subtraction of the resulting values from the corresponding stored part elevations (since the network is trained to reproduce point-wise differences between part and die). 
The neural network architecture employed in this experiment was a conventional Perceptron of four layers. The input layer comprised 21 non-bias nodes, the first hidden layer 13 non-bias nodes, the second hidden layer 3 nonbias nodes, and the output layer 1 non-bias node. Of the 21 input nodes, 19 were employed for representing Patch data, two for representing the values of the "material parameter" and "forming parameter" values stored with each data set. The network was trained according to the gradient-descent, backpropagation of error method. During early stages of training, the common practice was to process multiple networks simultaneously. When a clearly superior network emerged, the poorer members of the initial set of networks were excised. During final training, learning rate and momentum were slowly relaxed from initial values of .1 and .001 respectively to .001 and .00001 .

Figure $3 \mathrm{a}$ is the conventional scatter diagram and represents network training and testing results as a function of expected result. It is emphasized that network predictions for the single test case (4096 more points) are included in the scatter diagram (and, surprisingly, account for very little of the scatter). At first glance, it might appear that the trained behavior of the network is insufficiently accurate to be of much utility for die shape prediction. Figure $3 \mathrm{~b}$, a three-dimensional perspective representation of the network-predicted die, suggests otherwise.

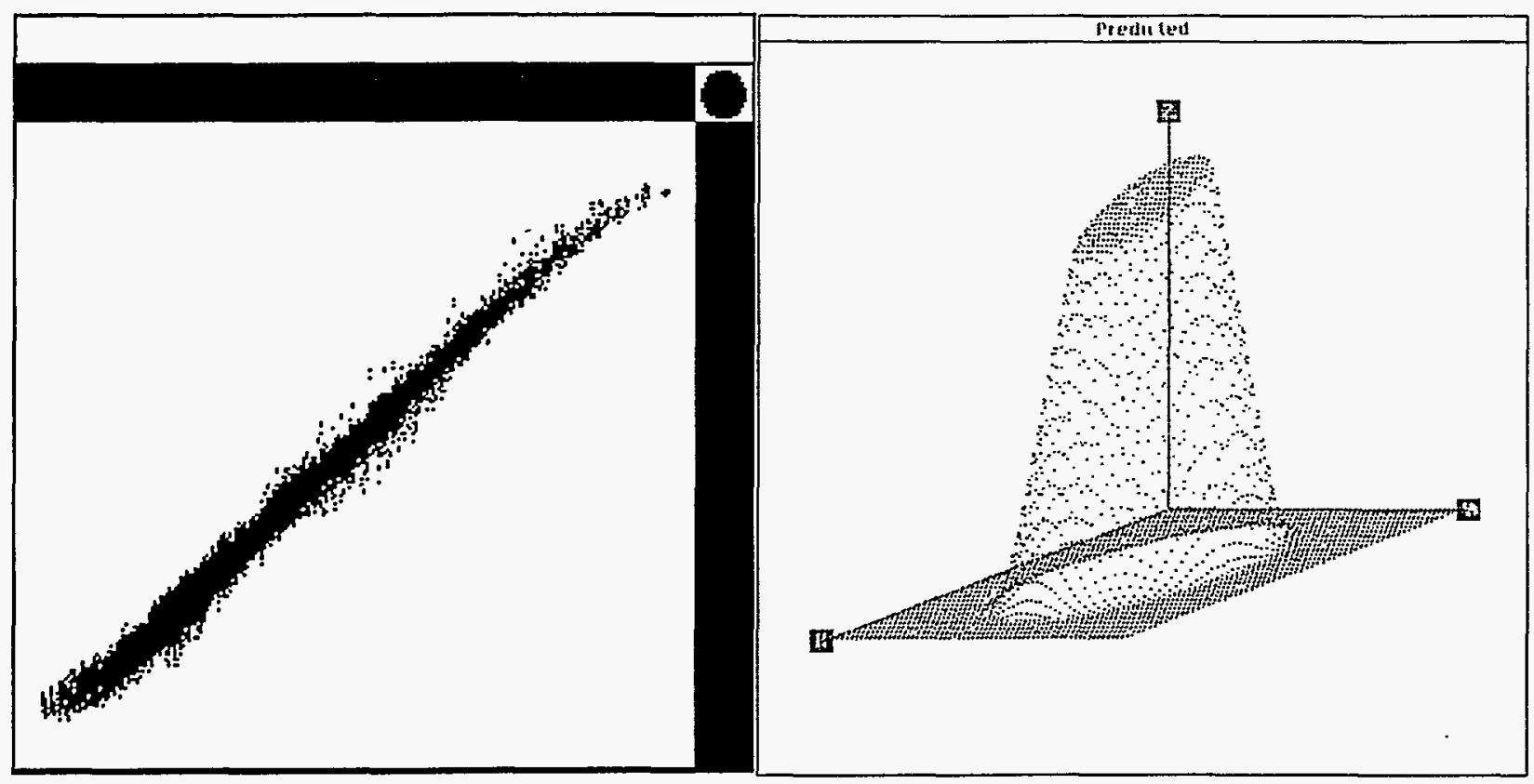

Fig. 3a. Scatter diagram for a training process (note that this includes 163840 points for the Pan and Shell training data and 4096 points for the single TopHat test case)

Fig. 3b. 3-D perspective plot of predicted die

Figure 4 provides a composite view of the Patch Method results and depicts the original part (dashes), the true die (i.e., the die from which the part was generated by the deformation model) (solid), the network-predicted die (dots) and the prediction error (times 10) for each point in the cross-section (crosses). In all cases, the prediction error has proved to be no greater than one part in 256 of the presented part elevation.

\section{SUMMARY}

In this paper, the development of, and experimental results of evaluating, a neural network-based system capable of inverse modeling of material deformation were described. Several alternative data representation methods were discussed. Of these, the Patch Method proved to have the most potential for accurate prediction of die geometries from user-supplied part shapes and was employed to obtain the presented experimental results in a demonstration of the potential utility of the methodology. 


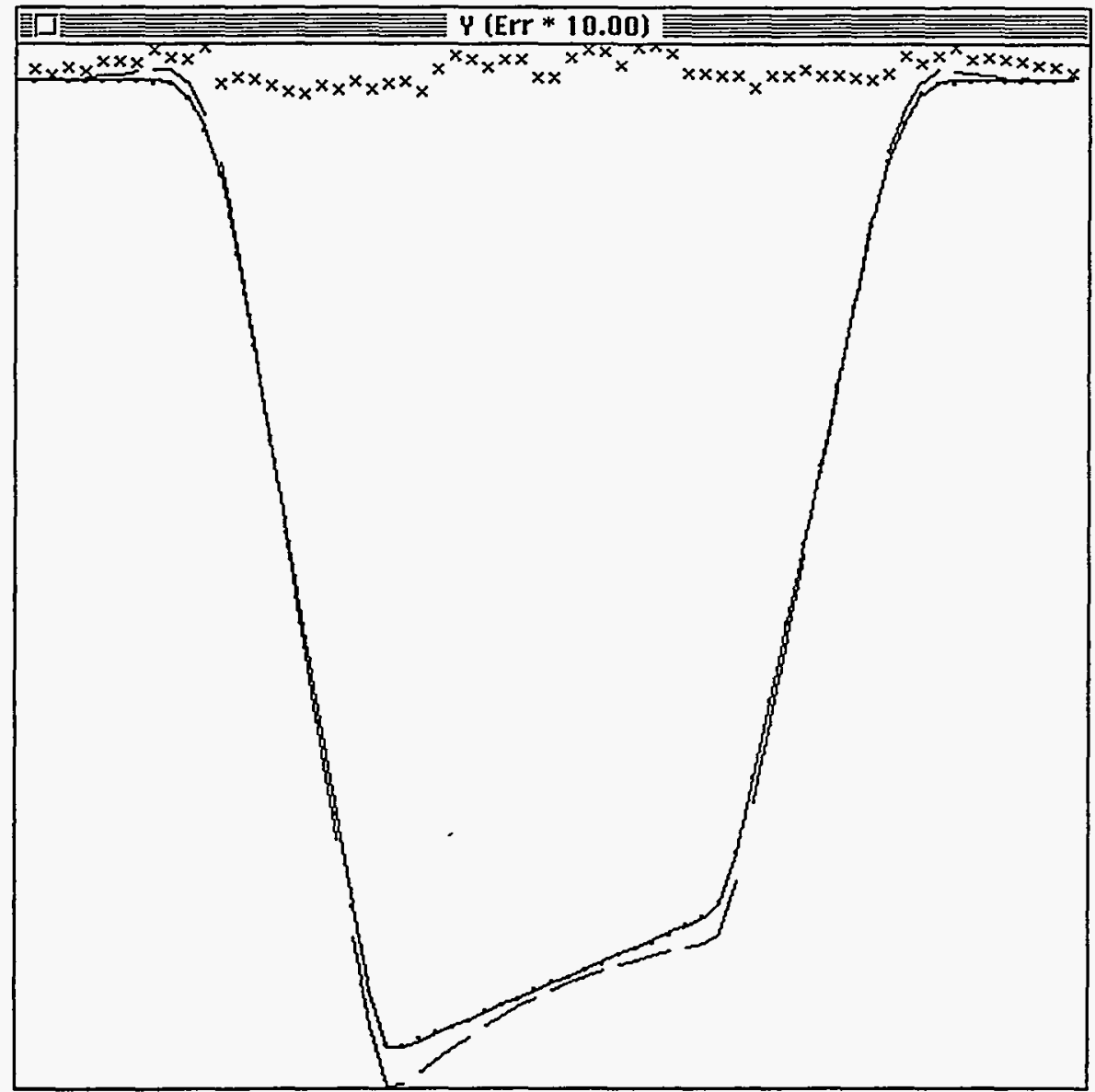

Fig. 4. Cross-section of part, (dashes), predicted die (dots), true die (solid), and die/true-die error X 10 (crosses)

\section{ACKNOWLEDGEMENTS}

This research was sponsored by the Laboratory Technology Research Program, Oak Ridge National Laboratory, managed by the Lockheed Martin Energy Research Corporation for the U. S. Department of Energy, under contract number DE-AC05-96OR22464. This submitted manuscript has been authored by a contractor of the U. S. Government under Contract No. DE-AC05-96OR22464. Accordingly, the U. S. Government retains a nonexclusive, royalty-free license to publish or reproduce the published form of this contribution, or allow others to do so, for U. S. Government purposes.

\section{REFERENCES}

1. S. Yang and K. Nezu, 1998. Application of an inverse FE approach in the concurrent design of sheet stamping. Journal of Materials Processing Technology, 79, 86-98.

2. B.T. Cheok and A.Y.C. Nee., 1998. Trends and developments in the automation of design and manufacture of tools for metal stampings. Journal of Materials Processing Technology, 75, 240-252.

3. N. Ivezic, J. H. Garrett, Jr., and R. Ganeshan, 1991. Generalized Hopfield Network for Structural Optimization. In Proceedings of Artificial Neural Networks in Engineering (ANNIE'91), St. Louis, Missouri, 849-854.

4. N. Ivezic and J. H. Garrett, Jr., 1994. A Neural Network-based Machine Learning Approach for Supporting Synthesis. Artificial Intelligence for Engineering Design, Analysis, and Manufacturing, 8, 143-161.

5. N. Ivezic and J. H. Garrett Jr., 1998. Machine learning for simulation-based support of early collaborative design. Artificial Intelligence for Engineering. Design, Analysis, and Manufacturing, 12, 123-139. 

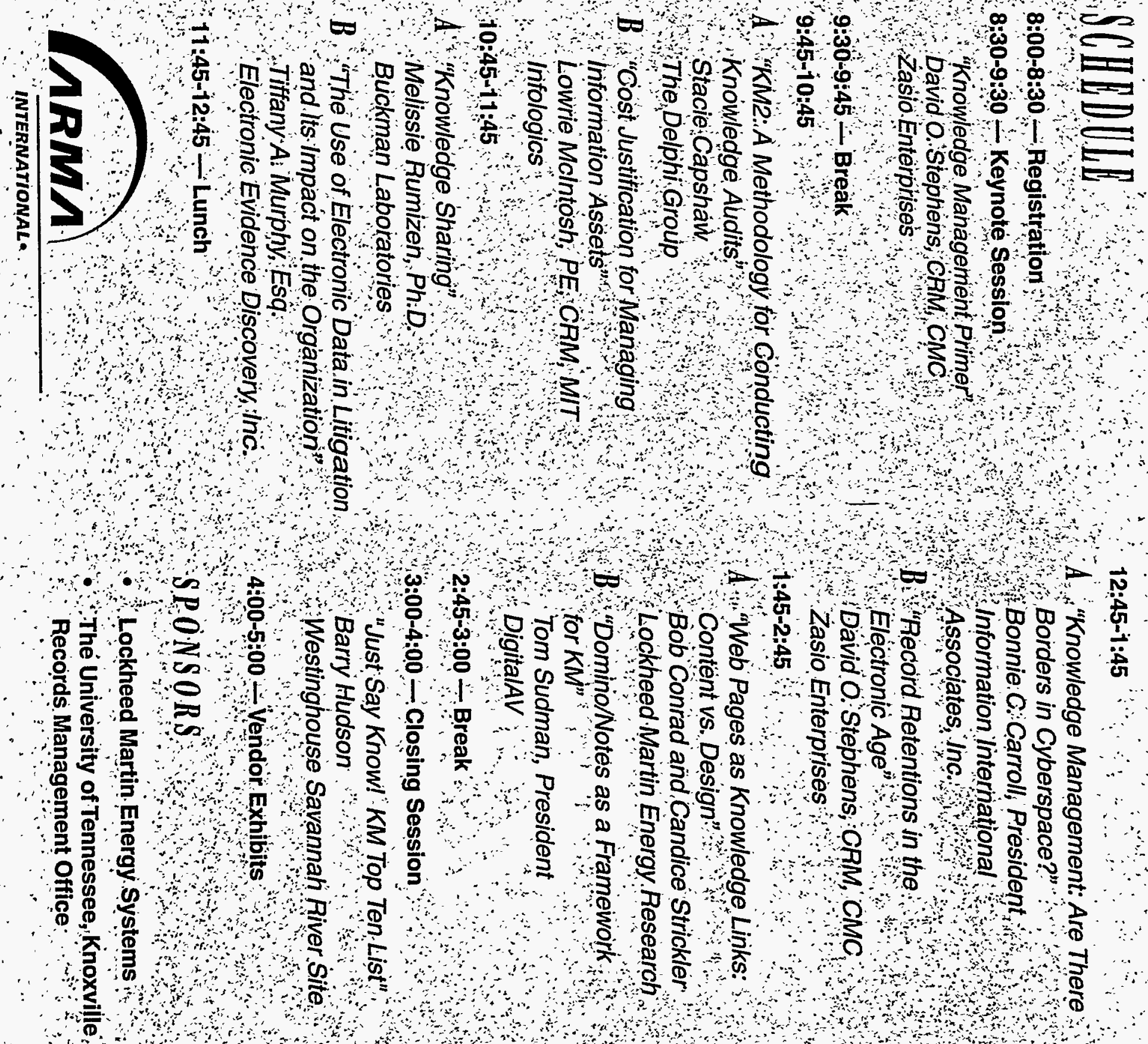

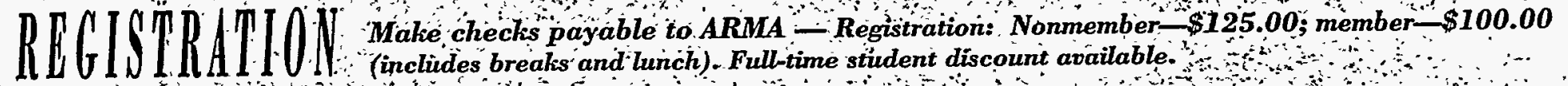

Name:

Company:

Address:

Phone:

Email:

Questions: Contact-Scott Troy

email szt@ornl.gov

423/574-0427 (phone)
Mail to: John Woodard

ARMA

1000 N. Central Street, Box 2

Knoxville, TN 37917 